\section{Retention of semantic, syntactic, and language information by young bilingual children*}

\author{
IVONNE HERAS and KEITH E. NELSON \\ Stanford University, Stanford, Calif. 94305
}

Bilingual 5-year-olds (20) were read stories in English and Spanish. Using variants of the second sentence in each story, recognition memory was tested for semantic and syntactic information. The children were also asked to identify the language of the third sentence in each story. The data indicate that the children successfully coded and remembered sentences in terms of meaning but rapidy forgot details of syntactic form. Coding by language (Spanish or English) for the third sentence was forgotten if followed by material in the altermate language.

How does the young child code the fully grammatical sentences of adults? This question is of interest in relation to a broad variety of developmental changes which depend upon the transmission of information between adults and children. In relation to language acquisition, the question seems critical. For in order to acquire the syntactic and semantic systems of a particular language, the child obviously must extract detailed information from well-formed sentences of the language.

Sachs (1967) has provided some interesting evidence that adults code sentences in terms of meaning but quickly forget syntactical details of a sentence once sentence meaning has been derived. She gave ner Ss passages of connected discourse. At the end of each passage, a recognition test sentence was presented. Some test sentences were identical to a sentence in the passage. some had been changed only in syntax, and some had been changed only in meaning. The amount of interpolated material between the original sentence and the test sentence was raried also. If the test sentence immediately followed the original sentence, Ss were very successful in recognizing changes in form as well as changes in meaning. But when the amount of interpolated material was increased to 160 syllables, changes in meaning were still recognized often, while recognition of changes in form dropped to about chance level.

In the present study, an adaptation of Sachs's technique was used to assess syntactic and semantic coding by bilingual 5-year-olds. In addition. we examined possible coding in terms of

* Appreciation for assistance goes to Dr. Guido Barrientos. Maureen Velarde. Hortencia Butler, Raquel Ruiz.. Jo Denham. Ruth Prehn. and Julie kirk. Thanks go also to Neal F. Johnson and to Rudolph W Schule for thoughtiul comments on a previous version of this vaper. While working on this studv, the first authur was recipient of a Dectoral Ford Futundation Fellowship for Mexican-American Students. the particular language, English or presented. Ss were read stories composed of sentences much like those in normal conversations between adults and 5-year-olds. After a delay produced by interpolated material, Ss were tested on particular sentences of each story. The following specific questions were of central concern: (1) Do children, like adults, recognize semantic changes more often than syntactic changes? (2) When the original and recognition test sentences are presented in different languages (English and Spanish). is recognition poorer than if both are presented in the same language? (3) Does the language of interpolated material affect a $S$ 's success in recalling the language (English or Spanish) in which a sentence was presented?

\section{METHOD}

The Ss were 20 children bilingual in English and Spanish. The mean age was 4-1 years, and ages ranged from 4-5 to 5.5 years. The S's bilingual abilities were determined from reports of nursery school teachers and from conversation with the $E$. For all $S$ s, Spanish was the predominant language, but both English and Spanish were spoken and comprehended well.

Each $S$ was tested individually in two $30-\mathrm{min}$ sessions, on consecutive mornings. The following instructions were given at the beginning of each session: "I'm going to tell you some stories that are lots of fun. They are in English and in Spanish. At the end of each story I'm going to say a sentence and you tell me if the sentence that I say was in the story or not. OK? I'm also going to ask you if the last sentence in the story was in English or in Spanish, OK?" The $S$ then was read one sample story. After the $S$ answered each of the two test questions following this story, the $\mathrm{E}$ explained the basis for the correct answer to the question. To insure that Spanish, in which a sentence was all Ss understood the task, Ss were given such an explanation for the sample story questions whether or not their own answers were correct.

In each session, 8 experimental stories followed the sample story and instructions. Each story was composed of a 5-word first sentence, a 7-word second sentence, and an 8-word third sentence. Two English and one Spanish sentence were employed in 8 of the 16 stores, while one English and two Spanish sentences were employed in the other 8 stories. Across stories, complete counterbalancing of the different possible sequences of English and Spanish sentences insured that $\mathrm{Ss}$ could not anticipate accurately whether a particular test sentence would be in Spanish or in English. Counterbalancing in terms of presentation order also occurred-in the first session, half of the Ss received Stories 1.8 and the remaining $S$ received Stories 9-16. As an attention-holding technique, at the beginning of each session the $\mathrm{S}$ was shown an incomplete picture (with stick-on decals) which was available for completion at the end of the session.

Immediately after each story, the $\mathrm{S}$ was read a test sentence and (in the same language as the test sentence) was asked if the test sentence was in the story. For eight of the stories, the test sentence was identical in meaning and syntax to the story's second sentence. For the remaining stories, the test senience-in relation to the story's second sentence-was either changed in syntax but not meaning or changed in meaning but not syntax. In order to avoid any extraneous clues useful for determining if a test sentence differed from a story's second sentence, two types of test sentences were used with equal frequency. "Type A" sentences were drawn from eight stories constructed so that the second sentence could be changed in meaning by changing both the subject noun and the verb while syntax remained the same. "Type B" sentences were drawn from eight stories constructed so that the second sentence could be changed in syntax by changing the position of a prepositional phrase while meaning was kept the same. From each of these sets of eight sentences, four were assigned randomly to an "identical" group and four to a "changed" group. Those test sentences in the changed group were then actually changed, while those in the identical group were not. Each semantically changed sentence was produced by replacing its original subject and verb with the original subject and verb of one of the other semantically changed sentences. Test sentences raried also in terms of 
language; eight of them matched in language the story's second sentence, and eight were changed in language (English-Spanish or Spanish-English). Thus, three within-Ss variables were studied for these test sentences based on Story Sentence 2: identical vs changed, type of sentence (A or $B$ ), and same language vs different language. These variables, in addition to the between-Ss factor of presentation order, were included in an analysis of variance performed on mean number of correct responses to test sentences.

For each story, the $\mathbf{S}$ also was asked whether the last (third) sentence in the story was presented in English or Spanish. This question was posed in the same language as the recognition test, which was interpolated between the third sentence and the question. In eight cases, the language used for this interpolated material was the same as the language used for the story's third sentence, and in eight cases the languages were different. These within-S factors of language for the interpolated material-English vs Spanish-and same or different language were included, together with presentation order, in an analysis of variance. The dependent variable was the mean number of times the Ss correctly identified the language of Story Sentence 3.

\section{RESULTS}

The Ss were much more successful in detecting semantic change rather than syntactic change. Number of correct responses per recognition test sentence averaged .825 for semantically changed Type A sentences, but only .363 for syntactically changed Type B sentences. In the latter case, Ss must have responded primarily on the basis of the unchanged meaning of the sentences. When Story Sentence 2 and the test sentence were identical, mean numbers correct for Sentence Type A (.888) and Sentence Type B (.863) did not differ significantly. Newman-Keuls multiple comparison tests indicated that the only significant differences between these four means were between the mean for syntactically changed sentences and each of the other three means (for each significant mean pair, $\mathrm{p}<.01)$. The interaction of Sentence Type by Identical-Changed was highly significant $(\mathrm{F}=23.34, \mathrm{df}=$ $1 / 126, \mathrm{p}<.001)$. Also significant were main effects of sentence type $(F=$ $28.99, \mathrm{df}=1 / 126, \mathrm{p}<.001)$ and identical-changed $(F=38.59, \mathrm{df}=$ $1 / 126, p<.001)$. As expected, Ss were slightly more successful when the test sentence and the story's second sentence were in the same language (mean $=.775)$ than when they were in different languages (mean $=.694$ ).
However, this difterence was not statistically significant $(F=3.22$, df $=$ $1 / 126, p<.10$ ). No other main effects or interactions approached significance.

For the third sentences of the stories, the children correctly identified the language of presentation more frequently when the sentence and interpolated material were in the same language (mean $=.700$ ) rather than in different languages (mean = .531 , chance level $=.500)$. This main effect was significant $(F=10.16, \mathrm{df}=$ $1 / 18, p<.01)$. No other main effects or interactions reached significance (in each case, $F \leqslant 2.92, \mathrm{df}=1 / 18$, p > .10).

\section{DISCUSSION}

The 5-year-olds in this study recognized changes in meaning far more often than they recognized changes in syntactic form. In fact, sentences that had been syntactically changed were judged as unchanged $64 \%$ of the time. Ss were apparently responding to most of these syntactically changed test sentences on the basis of unchanged sentence meaning. The simplest interpretation of these results is that the children derived the meaning of the original sentence and then rapidly forgot syntactic details of the sentence. This interpretation matches Sachs's (1967) interpretation of her finding, for adults tested with material interpolated between the original and test sentences, that syntactical changes were much more poorly recognized than were semantic changes. Despite the overall similarity in findings, however, the absolute level of recognition for syntactically changed sentences was lower in the present study than in Sachs's (1967) study. This was so despite the fact that the amount of interpolated material was higher in her study $(80$ or 160 syllables) than in the present study (an average of 12 syllables, a range of 9-17 syllables).

Performance in the present study was probably distorted in two ways from the child's typical use of language. As Ss were told that they would be asked about sentences at the end of each story, the extent to which meaning and syntax were remembered may have been maximized. On the other hand, performance may have been reduced because contextual cues, present in most natural situations, were not available. Nevertheless, the finding that the preschool child is sensitive to sem antic information-under circumstances when syntactic detail is lost or irrelevant--recurs in a variety of forms in both naturalistic and laboratory investigations (cf. Slobin, 1966; Brown \& Hanlon, 1970; Bever, 1970; Turner \&
Rommetveit, 1967; Smith, 1970 Bloom, 1970). 'The phenomenon most directly related to the present results is that children's reproductions of adult sentences often preserve meaning but assume new syntactic forms (Fraser Bellugi, \& Brown, 1963; Slobin \& Welsh, 1968).

Correct identifications of the language in which the third sentence was presented were well above chance in frequency only when the same language was used for presentation and for material interpolated between presentation and test. This observation suggests that $S$ s were coding the third sentence in terms of its English or Spanish presentation but that this coding was lost when the sentence was followed by material presented in the other language. For the second sentences of the stories, no direct test of the S's memory of the presentation language was provided. But if coding occurred in terms of presentation language, this coding did not significantly alter $S s^{\prime}$ recognition performance for the test sentences. Considering these findings on language together with the other results above, a broader conclusion is appropriate: sentence meaning was coded, and retained successfully, in a manner which was not dependent upon original syntactic form or original language.

We view the present findings as a departure point toward more differentiated analyses of sentence coding. The conclusion that syntactic but not semantic information is rapidly forgotten may or may not prove accurate for instances of semantic and syntactic change other than those examined in the present study and in Sachs's (1967) study. In any case, a differentiated analysis of the relative retention of semantic and syntactic information will require results based upon broader stimulus arrays. Also needed is the development of a common metric for measuring stimulus change. Given such a metric, Ss' recognition sensitivity to syntactic changes and semantic changes precisely equated in magnitude could be compared. An additional concern in future developmental work could profitably be the relationships between developmental changes in the child's coding of sentences and changes in the semantic and syntactic structure of sentences produced by the child.

\section{REFERENCES}

BEVER, T. A. The cognitive basis for linguistic structures. In J. R. Hayes (Ed.), Cognition and the development of language. New York: Wiley, 1970. Pp. 279-352.

BLOOM, L. Language development: Form and function in emerging grammars. 
Cambridge, Miss: M.1.T. Press, 1970.

BROWN, R.. \& HANLON, C. Derivational complexity and order of acquisition in child speech. In J. $R$. Hayes (Ed.) Cognition and the derelopment of language. New York: Wiley, 1970. Pp. 11-53.

FRASER, C., BEILUGI, U., \& BROWN, R Control of grammar in imitation, comprehension. and production. Journal of Verbal Learning \& Verbal Behavior. $1963,2,121-135$.
SACHS, J. S. Recognition memory for syntactic and semantic aspects of connected discourse. Perception \& Psychophysics, 1967,2, 437-442.

SLOBIN, I). I. Grammatical transformations and sentence comprehension in childhood and aduthood. Journal of Vertal Learning \& Verbal Behavior, 1966, 5. 219-227.

SLOBIN, D. I., \& WEI,SH, C. Elicited imitation as a research tool in developmental psycholinguistics.
Unpublished paper, Department of Psychology, University of California. Berkeley, 1967.

SMITH. C. An experimental approach to children's linguistic competence. In J. R. Hayes (Ed.), Cognition and the development of language. New York: Wiley, 1970. Pp. 109-135.

TURNER, F. A., \& ROMMETVEIT, R. The acquisition of sentence voice and reversibility. Child Development, 1967. $38,649-660$. 